\title{
Preoperative Evaluation of the Frail Patient
}

\author{
Lolita S. Nidadavolu, MD, PhD ${ }^{\star}$, April L. Ehrlich, MD, Frederick E. Sieber, MD ${ }^{\dagger}$, Esther S. \\ Oh, MD, PhD ${ }^{*}, \S, \S$
}

*Department of Medicine, Johns Hopkins University School of Medicine, Baltimore, Maryland; tDepartment of Anesthesiology and Critical Care Medicine, Johns Hopkins University School of Medicine, Baltimore, Maryland; ¥Department of Psychiatry and Behavioral Sciences, Johns Hopkins University School of Medicine, Baltimore, Maryland; §Department of Pathology, Johns Hopkins University School of Medicine, Baltimore, Maryland; "Johns Hopkins University School of Nursing, Baltimore, Maryland.

\section{Abstract}

Perioperative management of older adults is a complex field that is heavily influenced by the clinical heterogeneity of older adults. Frailty - a geriatric syndrome in which a patient is more vulnerable to stressors due to decreases in physical function and reserve- - has been indicative of adverse postoperative outcomes. Many tools have been developed to measure frailty that incorporate a variety of factors including physical and cognitive function, comorbidities, selfreported measures of health, and clinical judgment. Most of these frailty assessment tools are able to identify a subset of patients at risk of adverse outcomes including postoperative complications, longer hospital length of stay, discharge to a higher level of care, and mortality. Frailty assessment before surgical interventions can also guide discussions among patients, their families, anesthesiologists, and surgeons to tailor operative plans for patients to mitigate this increased risk. Studies are ongoing to identify interventions in frail patients that can improve postoperative outcomes, but high-quality data in the form of randomized controlled trials are lacking at this time.

In the United States, approximately one-third of all operating room-based procedures are performed on adults $\succeq 5$ years. ${ }^{1}$ Rates of surgical complications increase with age; in 1

Address correspondence to Esther S. Oh, MD, PhD, Division of Geriatric Medicine and Gerontology, Johns Hopkins University School of Medicine, Asthma and Allergy Center, 5501 Hopkins Bayview Circle, Room 1B.76, Baltimore, MD 21224.

eoh9@jhmi.edu.

DISCLOSURES

Name: Lolita S. Nidadavolu, MD, PhD.

Contribution: This author helped conceptualize, write, and revise the manuscript.

Name: April L. Ehrlich, MD.

Contribution: This author helped conceptualize, write, and revise the manuscript.

Name: Frederick E. Sieber, MD.

Contribution: This author helped conceptualize and revise the manuscript.

Name: Esther S. Oh, MD, PhD.

Contribution: This author helped conceptualize, write, and revise the manuscript.

This manuscript was handled by: Robert Whittington, MD.

The authors declare no conflicts of interest.

Supplemental digital content is available for this article. Direct URL citations appear in the printed text and are provided in the HTML and PDF versions of this article on the journal's website (www.anesthesia-analgesia.org). 
study of adults $>80$ years, $20 \%$ developed postoperative complications including pneumonia, prolonged ( $>48$ hours) ventilator support, and cardiac arrest. ${ }^{2}$ This has implications on the practice of anesthesia as the geriatric population has a unique physiology that affects their surgical outcomes. One such challenge is frailty. Frailty is a biologic syndrome characterized by decreased homeostatic reserve and diminished resistance to stressors due to cumulative declines across multiple physiologic systems that result in vulnerability to adverse outcomes. 3

Approximately 1 in 6 community-dwelling individuals $>60$ years may be frail, representing a significant portion of older individuals presenting for surgery. ${ }^{4}$ A prospective cohort study comparing 2 frailty assessment tools demonstrated that between $35 \%$ and $41 \%$ of patients were frail, and these frail patients were more likely to have adverse outcomes including more postoperative complications, increased length of stay (LOS), and higher 30-day readmission rates. ${ }^{5}$ Other studies showed that higher frailty scores were associated with higher risk of postoperative 30-day mortality after adjusting for age and American Society of Anesthesiology (ASA) classification. ${ }^{6,7}$ These studies demonstrate the importance of identifying frail older individuals who are planned for elective and emergent surgeries.

This narrative review will discuss different frailty assessment tools that have been validated in surgical populations and examine the association of preoperative frailty with postoperative outcomes. Although there are younger adults who are frail, this review will focus on older adults because frailty is strongly associated with increasing age and most of the validated tools have been extensively studied in older populations. ${ }^{8}$

\section{METHODS}

Electronic databases including PubMed, Embase, and the Cochrane Library were searched with date restrictions of January 1, 2001 to August 22, 2019. We started our search in 2001 due to the publication of the Physical Frailty Phenotype (PFP) and the Deficit Accumulation Index (DAI) frailty assessments in 2001. ${ }^{3,9}$ A combination of controlled vocabulary and keyword terms was used for the concepts of frail elderly, preoperative care, assessment, complications or outcomes, and survey instruments. A total of 1869 records were identified. Duplicate records were removed, and 1510 titles and abstracts were identified. Inclusion criteria are as follows: surgical population, age $\ 65$, frailty assessed by a validated tool, and reporting on postoperative complications and other clinical outcomes. Exclusion criteria are as follows: oncological surgeries or procedures, age $<65$, studies assessing different surgical techniques or approaches, studies focusing on the economic and financial impact of frailty, and conference abstracts. In addition, studies that did not utilize all components of previously validated frailty tools (eg, only using gait speed or handgrip strength) were excluded. We also reviewed reference lists in relevant review articles to identify additional studies. Articles were reviewed by L.S.N. and A.L.E., and consensus was reached for final inclusion of eligible studies. Of the 1869 articles initially identified, 32 articles met all inclusion and exclusion criteria (Supplemental Digital Content, Figure, http:// links.lww.com/AA/D38). 


\section{RESULTS}

\section{Frailty Assessment Tools}

Conceptual Frameworks Behind Frailty Assessment Tools.-There are 2 major conceptual frameworks behind the most commonly used frailty measurement tools. ${ }^{10}$ The first is the concept of frailty as a biological syndrome characterized by energy depletion as exemplified by the PFP (Table 1). The PFP takes into account physical measurements of grip strength and walking speed and raises questions about exhaustion, physical activity, and unintentional weight loss as key clinical presentations of decreased physiologic reserve. ${ }^{3}$ The 5 components of the PFP are considered to be proxy measurements of dysregulation in stress response and energy metabolism. ${ }^{10}$

The second framework for defining frailty is considering frailty as an accumulation of deficits across functional, physical, cognitive, and social measures. ${ }^{10}$ Instruments that define frailty as "deficit accumulation" include the DAI, the Johns Hopkins Adjusted Clinical Groups (JHACG) frailty assessment, and the modified Frailty Index 11 (mFI 11). ${ }^{9,21,22}$ These tools measure factors including nutrition, comorbidities, functional status, disability, and mental health, and a cumulative score is derived. Similarly, the Brief Frailty Instrument by Rockwood ${ }^{15}$ incorporates an operational definition of frailty beyond measuring activities of daily living by including cognitive impairment and incontinence components to their tool. The JHACG frailty assessment and DAI utilize specific diagnosis codes or the presence/ absence of specific comorbidities to define a frail subset of patients (Table 1) ${ }^{9}{ }^{921}$ Both the PFP and deficit accumulation models, such as the DAI and mFI 11, are more predictive of a patient's cumulative risk of adverse outcomes than age alone. ${ }^{10}$

Measurement Issues in Frailty Assessment Tools.-Although there is a range of factors that comprise different frailty assessment tools, 2 domains (ie, physical and cognitive function) that may require active measurement are discussed below.

Physical Measures.: The 2 physical measurements in the PFP—gait speed and handgrip strength-require training to standardize measurements. A comprehensive review of handgrip strength measurement demonstrates that factors-model of dynamometer, posture and arm position of patient, and handle position—can influence results and make it challenging to compare results when different protocols are used. ${ }^{25}$ Gait speed measurement and Timed Up and Go (TUG) testing requires a dedicated location where 4- and 3-m markings can be placed on the floor for the respective tests. ${ }^{24,26}$ This requirement for specialized equipment and space may be challenging for some outpatient preoperative clinics or in the home of a patient. In addition, patients with limited mobility due to pain may find their preoperative gait speed and TUG measurements to be an inaccurate assessment of their premorbid functioning.

Cognitive Assessments.: Underlying cognitive impairment is a well-known risk factor for many different postoperative complications including delirium. In 1 recent study of over 7800 patients undergoing hip fracture repair, dementia diagnosis was one of the strongest risk factors for postoperative delirium. ${ }^{27}$ Patients identified as having mild cognitive impairment (MCI) before surgery were also at higher risk of developing postoperative 
delirium, and demonstrated increased risk of adverse outcomes, specifically higher rates of discharge to a postacute facility and new impairment in cognitive instrumental activities of daily living (IADL) 1 month later. ${ }^{28} \mathrm{~A}$ study of cognitively impaired patients undergoing vascular surgery demonstrated significantly higher rates of wound infections and longer LOS (>10 days) compared to cognitively normal patients. ${ }^{29}$ Furthermore, patients with dementia are at higher risk of mortality after surgery compared to patients with normal cognition (hazard ratio $[\mathrm{HR}]=1.84 ; 95 \%$ confidence interval $[\mathrm{CI}], 1.10-3.07$ ). ${ }^{30}$

Despite the strong association of underlying cognitive function with poor postoperative outcomes, not all frailty assessment tools incorporate cognitive assessment. The PFP; Vulnerable Elders Survey (VES); Fatigue, Resistance, Ambulation, Illnesses, Weight Loss (FRAIL) Scale; and mFI 11 do not have an explicit component of preoperative cognitive assessment built into their assessment tools. The DAI and JHACG rely on self-report of cognitive impairment, and the Edmonton Frail Scale (EFS) has a limited active cognitive screening component (eg, clock draw). Active measurement of cognitive function is important as these measures may be used to determine a patient's response to perioperative stressors and could allow monitoring of longitudinal cognitive trajectory. ${ }^{31}$

With respect to the feasibility of incorporating cognitive assessment into an outpatient preoperative visit, the Montreal Cognitive Assessment (MoCA)—a well-validated study with high reliability—can be completed in approximately 10 minutes. ${ }^{29} \mathrm{~A}$ review by Long et $\mathrm{al}^{32}$ details several other short ( $\mathcal{\Omega} .5$ minutes) cognitive assessments that can be completed preoperatively including the Mini-Cog, which consists of a 3-word recall and clock drawing test. Incorporating cognitive testing in a preoperative clinic has been shown to be feasible and not burdensome to either practitioners or patients. ${ }^{33}$ Due to the lack of active cognitive screening component in most frailty assessment tools, it would be important to incorporate an additional cognitive screening measure into the peri-operative workflow.

Time to Complete Frailty Assessments.-One of the challenges of preoperative evaluation for frailty is the length of time to perform such assessments in a busy outpatient clinic. ${ }^{10}$ In general, newer frailty assessment tools are designed to be shorter in duration and more conveniently performed in an outpatient preoperative clinic (Table 1). At this time, tools such as the PFP require patient participation, specialized equipment, staff training for standardized assessment, and additional time to perform the physical measurements. Simpler versions of existing frailty tools continue to be developed (eg, the modified Frailty Index 5 [mFI 5]). MFI 5 uses a subset of measures from the mFI 11 and has also been shown to have good sensitivity for identifying frail older adults preoperatively. ${ }^{22,34}$ Frailty assessment tools, such as mFI 11 and JHACG, are further streamlined by relying on data from electronic medical records or the American College of Surgeons National Surgical Quality Improvement Program (ACS-NSQIP) database. Relevant medical comorbidities, changes in weight, and baseline physical and cognitive function can be identified and used in these frailty assessment tools without relying solely on patient self-report. 


\section{Postoperative Outcomes Associated With Frailty}

Frail patients overall have worse health care outcomes (eg, postoperative complications, LOS, mortality) compared to nonfrail counterparts in both elective and emergent surgical procedures (Table 2). Frail and nonfrail patients had similar surgical procedures in these studies, with exception of vascular procedures, in which frail patients primarily underwent peripheral vascular interventions and were less likely to have major elective vascular surgery than nonfrail patients. ${ }^{47} \mathrm{~A}$ study examining general, vascular, cardiac, thoracic, and orthopedic surgeries in a large population showed that the most robust patients (Risk Analysis Index $[\mathrm{RAI}] \leq 10$ ) comprised $80 \%$ of the surgical population, while frail patients (RAI 221 ) comprised only $7 \%$ of the population. ${ }^{11}$ As frail patients constitute approximately $15 \%$ of community-based individuals who are over 65 years of age, ${ }^{62}$ there may be a selection bias where poor operative candidates are excluded from undergoing surgery. ${ }^{63}$ This discor-dance between the percentage of frail patients in the overall population compared to the percentage of frail patients undergoing major surgical procedures suggests that surgical studies are likely to underestimate postoperative risk in frail patients.

Postoperative Complications.-Overall, higher postoperative complication rates were associated with frailty status (Table 2). A study examining emergency general surgeries by Joseph et al ${ }^{36}$ showed that major complication (eg, sepsis, pneumonia, deep venous thrombosis) rates in frail patients were $>3$ times more likely than in robust patients (odds ratio $[\mathrm{OR}]=3.87 ; 95 \% \mathrm{CI}, 1.69-8.84)$. All 4 studies examining patients undergoing orthopedic surgeries (elective and hip fracture repairs) demonstrated higher rates of complications for patients categorized as frail as well. ${ }^{5,46,49,55}$ Although many of the studies did not examine delirium as one of the main postoperative complications, 1 study by Gleason et al ${ }^{49}$ demonstrated that postoperative delirium rates were higher in frail compared to nonfrail patients undergoing orthopedic procedures (28\% vs $3.4 \%, P=.01)$.

In terms of frailty tool-specific findings, frailty status as defined by the DAI, Groningen Frailty Indicator (GFI), VES, and Clinical Frailty Scale (CFS) was associated with higher rates of postoperative complications in patients undergoing emergency surgery $35,36,43,44$; however, this was not observed with the Brief Frailty Instrument. ${ }^{35}$ Interestingly, the VES and GFI were used alongside the Brief Frailty Instrument in the same surgical population to categorize frailty status. Frailty identified by VES and GFI was associated with higher rates of complications in frail patients suggesting that the Brief Frailty Instrument may underestimate risk of postoperative complication in this population. ${ }^{35}$ A meta-analysis examining $\mathrm{mFI} 11$ use across various surgical settings showed higher risk of all complications (relative risk $[\mathrm{RR}]=1.48 ; 95 \% \mathrm{CI}, 1.35-1.61$ ), Clavien-Dindo Class IV complications (requiring critical care management) $(\mathrm{RR}=2.03$; 95\% CI, 1.26-3.29), and wound complications ( $\mathrm{RR}=1.52 ; 95 \% \mathrm{CI}, 1.47-1.57)$ for frail patients compared to robust patients. ${ }^{54}$

A large retrospective study by Shah et $\mathrm{al}^{54}$ using data from ACS-NSQIP demonstrated that frailty is associated with higher complication rates even in low-risk procedures (average 30day mortality rate $\unlhd \%$ ). In this study, the proportion of individuals with any complication 
was higher in the most frail group (Clinical Risk Analysis Index [RAI-C] >40) compared to the robust group (RAI-C $\leq 10)$ undergoing low-risk procedures $(42.9 \%$ vs $4.4 \%, P<.001)$. Findings were similar for major complications in the same population and with higher proportion of individuals with major complication in the most frail group (RAI-C $>40$ ) compared to the robust group (RAI-C $\leq 0)(36.4 \%$ vs $3.2 \%, P<.001)$. Both of these findings suggest a dose- response association between the degree of frailty and the number of postoperative complications. ${ }^{11}$

Length of Stay.-Frailty was significantly associated with LOS in 20 of 22 studies (Table 2). Two studies that did not show significant association of frailty with LOS examined elective general surgery and vascular procedures and, in general, had smaller samples compared to the studies that showed significant associations. ${ }^{12,38}$ One study using 2 different frailty assessment tools in elective total joint replacement patients showed that the frailty status categorized by the CFS was significantly associated with LOS while those categorized by FRAIL scale was not. ${ }^{42}$ The CFS is based on the deficit accumulation model, 18 and the FRAIL scale is based more on Fried's phenotypic model. ${ }^{20,42}$ The authors postulated that their study population, which consisted of elective orthopedic surgery patients, may have excluded more frail patients (eg, the hip fracture population). ${ }^{42}$

Factors that could be contributing to increased LOS for frail patients are increased postoperative complication rate and additional time required to arrange discharge to skilled facility. Among the orthopedic surgeries that examined LOS as an outcome, 7 of 8 studies demonstrated significantly increased LOS in frail patients. Frailty assessment tools used in these studies ranged from DAI, PFP, CFS, FRAIL scale, and mFI. In general, most studies examined LOS as a continuous variable. Gleason et al ${ }^{49}$ examined LOS as both a binary (> or $\mathbf{6}$ days) and a continuous variable and found statistically significant associations of higher frailty score with longer LOS in both analyses.

The study by Drudi et $\mathrm{al}^{38}$ demonstrated association of frailty with mortality and worsening disability after interventions for peripheral arterial disease (PAD) but not with LOS. The study by Vernon et al ${ }^{12}$ using the VES in patients undergoing elective surgical procedures examined LOS as a binary variable to primarily observe whether patients required noninvasive positive pressure ventilation following their procedure or an unanticipated hospital stay. However, the authors state that their study was significantly limited by missing data, with only 48 of 103 patients having complete surveys from which the researchers could calculate frailty score. Smaller sample size may have contributed to the nonsignificant findings between frailty status and LOS in this study. ${ }^{12}$

Postoperative Mortality.-Twenty-five studies specifically examined associations between frailty status and 30-day mortality. One study by McIsaac et al ${ }^{50}$ examining independent, community-dwelling patients demonstrated that mortality was particularly high among frail patients in the immediate postoperative period. In this study, frail patients undergoing emergency surgical procedures were 23 times as likely as nonfrail patients to die on postoperative day $1(\mathrm{HR}=23.1 ; 95 \% \mathrm{CI}, 22.3-24.1) .{ }^{50}$ Among patients undergoing major elective noncardiac surgery, 1-year mortality remained increased for frail versus nonfrail patients even after adjusting for demographics, procedure types, and 19 different 
medical comorbidities (HR $=1.36,95 \% \mathrm{CI}, 1.26-1.46) .{ }^{51}$ Other studies of patients undergoing emergency procedures demonstrated similar increases in rates of postoperative mortality for frail patients. ${ }^{35,36,43,44}$ Four of the 25 studies did not show statistically significant associations between frailty and postoperative mortality. ${ }^{37,38,46,49}$ These studies had smaller sample sizes and included patients undergoing hip fracture repair, general orthopedic surgeries, and vascular surgery. ${ }^{38,46}$ One study by Hall et $\mathrm{al}^{23}$ examining elective surgery patients showed, for those determined to be frail, that mortality 1 year following surgery remained elevated compared to the overall 1 -year mortality for the group (26.8\% vs $3.5 \%)$.

\section{Postoperative Discharge Disposition and 30-Day Readmission Rates.-A}

majority of the results from Table 2 demonstrate that, depending on the type of surgery, frail patients are at higher risk of functional impairment from prolonged LOS or complications than a nonfrail patient. These adverse outcomes can lead to patients requiring discharge to subacute nursing facilities for continued care. One study found that frail vascular surgery patients were 1.6 times as likely as robust patients to be discharged to a postacute care facility instead of their homes $(\mathrm{OR}=1.6 ; 95 \% \mathrm{CI}, 1.4-1.8) .{ }^{56}$ Orthopedic surgery studies examining discharge disposition demonstrated that the PFP, DAI, CFS, and $\mathrm{mFI}, 5,42,55$ but not the FRAIL scale, were associated with increased rates of discharge to skilled facilities in this population. ${ }^{42}$ Among community-dwelling older adults undergoing emergency surgery, frail patients were 5.8 times as likely as robust patients to be discharged to a postacute care facility (OR $=5.8 ; 95 \% \mathrm{CI}, 5.53-6.12) .{ }^{50}$ A meta-analysis looking at the use of $\mathrm{mFI} 11$ in surgical patients similarly showed increased relative risk of discharge to postacute care following surgery for frail patients compared to robust patients. ${ }^{54}$ The frailty assessment tools that demonstrate positive associations between frailty and 30-day readmission are the $\mathrm{mFI}$ and RAI-C, examining general surgery, total hip arthroplasty, and general urological procedures $^{54,55,60}$ (Table 2).

\section{Comparison of Frailty Tools to Other Surgical Risk-Stratifying Tools.-Frailty} assessment tools have been compared to other tools assessing surgical risk (eg, ASA classification and comorbidity index). One study looking at mFI 11 in total hip arthroplasty population showed that $\mathrm{mFI} 11$ was a stronger predictor of readmission rates $(\mathrm{OR}=14.72$; 95\% CI, 6.95-31.18) than the ASA classification or age. ${ }^{55}$ MFI 11 was also a better predictor of any complication $(\mathrm{OR}=3.63 ; 95 \% \mathrm{CI}, 1.64-8.05)$ and reoperation $(\mathrm{OR}=6.52$; 95\% CI, 2.48-17.13), while ASA classification and age did not show statistically significant association with these outcomes. ${ }^{55}$ Other studies showed that frailty was predictive of higher risk for 30-day mortality and discharge to a skilled facility, while age and ASA score were not associated with these outcomes. ${ }^{36,47}$ However, 1 study found that addition of frailty measurement to ASA class improved the ability to predict postoperative complications and discharge to a skilled nursing facility. ${ }^{39}$

\section{DISCUSSION}

Preoperative assessment of frailty using tools validated for surgical populations is one of the first steps in identifying patients who are at high risk for poor postoperative outcomes. Across different surgical populations, frailty is associated with greater overall postoperative 
complications, longer hospital LOS, and higher mortality. Therefore, preoperative knowledge of frailty can help guide discussions with the patient's care team to optimize perioperative care.

There is no gold standard assessment for frailty, particularly among older individuals undergoing surgery. Assessment tools vary in the domains assessed (ie, cognition, comorbidities, and physical function), source of information (ie, direct assessment, selfreport, and electronic health records), time required, and location of evaluation (ie, outpatient, inpatient, and by telephone). In considering the data presented in Tables 1 and 2, frailty assessment tools most predictive of postoperative complications, longer LOS, and higher mortality include the mFI, CFS, PFP, and RAI-C. The mFI and RAI-C, in particular, can pull in the relevant information needed to assess frailty from a patient's electronic medical records. The PFP can also be a useful frailty assessment tool in a preoperative clinic, particularly once staff is trained on standardized measurement of grip strength and gait speed. In addition, the PFP also has a well-characterized biologic framework and animal models in which proposed interventions for frailty can be tested for efficacy. ${ }^{64,65}$

The feasibility of implementing frailty assessment in a preoperative clinic visit is an important consideration. Recently developed tools (mFI 11, CFS, RAI-C, and the FRAIL scale) can be completed in approximately 10 minutes without requiring physical measures (eg, hand grip or timed walk). ${ }^{13}$ The VES has also been successfully administered over the telephone. ${ }^{12}$ With the right tools and appropriate training, both PFP and DAI can be performed in a clinic setting. 5,66

It is less clear what should be done once a patient is identified as frail and is scheduled to undergo an elective or emergent surgical procedure. Some strategies that can be implemented for both frail and nonfrail patients include preoperative optimization of comorbidities such as diabetes, hypertension and congestive heart failure, nutritional assessment, and closer postoperative monitoring in a stepdown unit. ${ }^{67}$ Geriatrics comanagement and consultation can also be implemented early on in a frail patient's hospital course. In the preoperative setting, identifying a frail patient should initiate further discussions of goals of care, and more comprehensive geriatric assessment (CGA) can be performed to identify medical, physical, or socio-economic vulnerabilities that contribute to a patient's frail status that can be targeted for intervention.

Several recent editorials describe a role for rapid frailty assessment tools with high negative predictive values to rule out frailty in surgical candidates, while allowing for patients who screen positive for frailty to undergo more rigorous preoperative assessments. ${ }^{31,68}$ These assessments may include a CGA, an interdisciplinary approach that systematically evaluates physical, functional, cognitive, environmental, and social domains for an older adult. ${ }^{69}$ An individualized treatment plan resulting from concerted interdisciplinary effort is the goal of a successful CGA. ${ }^{70}$

An example of what can be done for individuals who are identified as frail is highlighted in study by Hall et al ${ }^{58}$ using the RAI-C and measuring the effectiveness of a "frailty screening initiative." This initiative consisted of clarifying goals of care and postoperative expectations 
for patients as well as informing the patient's surgeons, anesthesiologists, and critical care physicians of the patient's frailty status. ${ }^{58}$ Overall 30 -day mortality for all subjects in the frailty screening initiative arm, regardless of frailty status, decreased from $1.6 \%$ preinitiative to $0.7 \%$ following the initiative, and frail subjects had the greatest reduction in 30-day mortality compared to robust patients, decreasing from $12.2 \%$ to $3.8 \% .58$

One intervention for frail surgical patients that is receiving more attention is prehabilitation. Prehabilitation is a multimodal intervention that aims to reduce vulnerability and increase resilience of patients to stressors such as surgery or nonsurgical interventions. ${ }^{67}$ Previous literature has considered prehabilitation as an intervention to reduce disability and restore function among frail patients before development of acute illness, injury, or surgery. ${ }^{71}$ However, there is no consensus for the optimal type of prehabilitation for frail patients, likely from the lack of robust randomized control trials at this time.

The strengths of this narrative review include a comprehensive evaluation of frailty assessment tools that were specifically validated in surgical populations as well as postoperative outcomes that have been examined among individuals who were identified as frail using these tools. Limitations of this review include focus on adults over age 65 . There are studies examining frail patients who are $<65$, particularly in cancer, cardiac surgery, and transplant medicine fields that were excluded based on our criteria. An additional limitation is that studies looking at oncologic surgeries and those with concurrent or pre-ceding chemotherapy were also excluded. The presence of cancer and chemotherapy can be an additional stressor to older adults, and patients with a history of cancer have significantly higher rates of frailty and vulnerability. ${ }^{72}$ A comprehensive review focusing on frailty in the oncologic setting has been recently published. ${ }^{73} \mathrm{We}$ also excluded studies that focused only on preoperative sarcopenia, but a review on the topic of sarcopenia and surgical outcomes has also been published recently. ${ }^{74}$

Determining a patient's preoperative frailty status is critical to assessing a patient's overall perioperative risk including postoperative complications, increased mortality, longer hospital LOS, and higher level of care on discharge. Preoperative knowledge of frailty status can guide discussions among patients, their families, anesthesiologists, and surgeons to tailor perioperative care for patients to mitigate this increased risk. All the frailty assessment tools identified in this review demonstrated the ability to identify frail individuals who were at higher risk of worse postoperative outcomes compared to nonfrail individuals in different surgical settings. These assessment tools incorporate different measurements that can be done in the outpatient setting or in the hospital before surgery, demonstrating feasibility. Ongoing studies on the efficacy of preoperative interventions targeting frail patients will be instructive in providing more information about how to best improve postoperative outcomes for frail older adults.

\section{Supplementary Material}

Refer to Web version on PubMed Central for supplementary material. 


\section{ACKNOWLEDGMENTS}

We would like to thank Carrie Price, MLS of Johns Hopkins University, Baltimore, MD, for her assistance with the literature search.

Funding: This study was funded by R01AG057725 (E.S.O.), the Roberts Family Fund (E.S.O.), and National Institute on Aging (T32AG058527) Translational Aging Research Training Program (L.S.N.).

\section{GLOSSARY}

ACS-NSQIP

ADL

ASA

CFS

CGA

CI

COPD

DAI

EFS

FRAIL

GFI

HR

IADL

ICU

JHACG

LOS

MCI

mFI 5

mFI 11

MI

MoCA

NS

OR
American College of Surgeons National Surgical Quality Improvement Program

activities of daily living

American Society of Anesthesiology

Clinical Frailty Scale

comprehensive geriatrics assessment

confidence interval

chronic obstructive pulmonary disease

Deficit Accumulation Index

Edmonton Frail Scale

Fatigue, Resistance, Ambulation, Illnesses, Weight Loss

Groningen Frailty Indicator

hazard ratio

instrumental activities of daily living

intensive care unit

Johns Hopkins Adjusted Clinical Groups

length of stay

mild cognitive impairment

modified Frailty Index 5

modified Frailty Index 11

myocardial infarction

Montreal Cognitive Assessment

not significant

odds ratio 


$\begin{array}{ll}\text { PAD } & \text { peripheral arterial disease } \\ \text { PFP } & \text { Physical Frailty Phenotype } \\ \text { RAI } & \text { Risk Analysis Index } \\ \text { RAI-C } & \text { Clinical Risk Analysis Index } \\ \text { RR } & \text { relative risk } \\ \text { TIA } & \text { transient ischemic attack } \\ \text { TUG } & \text { Timed Up and Go } \\ \text { VES } & \text { Vulnerable Elders Survey }\end{array}$

\section{REFERENCES}

1. Elixhauser A, Andrews RM. Profile of inpatient operating room procedures in US hospitals in 2007. Arch Surg. 2010;145:1201-1208. [PubMed: 21173295]

2. Hamel MB, Henderson WG, Khuri SF, Daley J. Surgical outcomes for patients aged 80 and older: morbidity and mortality from major noncardiac surgery. J Am Geriatr Soc. 2005;53:424-429. [PubMed: 15743284]

3. Fried LP, Tangen CM, Walston J, et al.; Cardiovascular Health Study Collaborative Research Group. Frailty in older adults: evidence for a phenotype. J Gerontol A Biol Sci Med Sci. 2001;56:M146M156. [PubMed: 11253156]

4. Ofori-Asenso R, Chin KL, Mazidi M, et al. Global incidence of frailty and prefrailty among community-dwelling older adults: a systematic review and meta-analysis. JAMA Netw Open. 2019;2:e198398. [PubMed: 31373653]

5. Cooper Z, Rogers SO Jr, Ngo L, et al. Comparison of frailty measures as predictors of outcomes after orthopedic surgery. J Am Geriatr Soc. 2016;64:2464-2471. [PubMed: 27801939]

6. Arya S, Kim SI, Duwayri Y, et al. Frailty increases the risk of 30-day mortality, morbidity, and failure to rescue after elective abdominal aortic aneurysm repair independent of age and comorbidities. J Vasc Surg. 2015;61:324-331. [PubMed: 25312534]

7. Farhat JS, Velanovich V, Falvo AJ, et al. Are the frail destined to fail? Frailty index as predictor of surgical morbidity and mortality in the elderly. J Trauma Acute Care Surg. 2012;72:1526-1530. [PubMed: 22695416]

8. Richards SJG, Frizelle FA, Geddes JA, Eglinton TW, Hampton MB. Frailty in surgical patients. Int J Colorectal Dis. 2018;33:1657-1666. [PubMed: 30218144]

9. Mitnitski AB, Mogilner AJ, Rockwood K. Accumulation of deficits as a proxy measure of aging. ScientificWorldJournal. 2001;1:323-336. [PubMed: 12806071]

10. Walston J, Bandeen-Roche K, Buta B, et al. Moving frailty toward clinical practice: NIA intramural frailty swcience symposium summary. J Am Geriatr Soc. 2019;67:1559-1564. [PubMed: 31045254]

11. Shah R, Attwood K, Arya S, et al. Association of frailty with failure to rescue after low-risk and high-risk inpatient surgery. JAMA Surg. 2018;153:e180214. [PubMed: 29562073]

12. Vernon TL III, Rice AN, Titch JF, Hill BF, Muckler VC. Implementation of Vulnerable Elders Survey-13 frailty tool to identify at-risk geriatric surgical patients. J Perianesth Nurs. 2019;34:911-918.e2. [PubMed: 30910510]

13. Bentov I, Kaplan SJ, Pham TN, Reed MJ. Frailty assessment: from clinical to radiological tools. $\mathrm{Br}$ J Anaesth. 2019;123:37-50. [PubMed: 31056240]

14. Kellen E, Bulens P, Deckx L, et al. Identifying an accurate pre-screening tool in geriatric oncology. Crit Rev Oncol Hematol. 2010;75:243-248. [PubMed: 20060313] 
15. Rockwood K A brief clinical instrument to classify frailty in elderly people. Lancet. 1999;353:205-206. [PubMed: 9923878]

16. Steverink N Measuring frailty: developing and testing the GFI (Groningen Frailty Indicator). Gerontologist. 2001;41:236.

17. Saliba D, Elliott M, Rubenstein LZ, et al. The vulnerable elders survey: a tool for identifying vulnerable older people in the community. J Am Geriatr Soc. 2001;49:1691-1699. [PubMed: 11844005]

18. Rockwood K, Song X, MacKnight C, et al. A global clinical measure of fitness and frailty in elderly people. CMAJ. 2005;173:489-495. [PubMed: 16129869]

19. Rolfson DB, Majumdar SR, Tsuyuki RT, Tahir A, Rockwood K. Validity and reliability of the Edmonton Frail Scale. Age Ageing. 2006;35:526-529. [PubMed: 16757522]

20. Abellan van Kan G, Rolland YM, Morley JE, Vellas B. Frailty: toward a clinical definition. J Am Med Dir Assoc. 2008;9:71-72. [PubMed: 18261696]

21. Sternberg SA, Bentur N, Abrams C, et al. Identifying frail older people using predictive modeling. Am J Manag Care. 2012;18:e392-e397. [PubMed: 23145847]

22. Velanovich V, Antoine H, Swartz A, Peters D, Rubinfeld I. Accumulating deficits model of frailty and postoperative mortality and morbidity: its application to a national database. J Surg Res. 2013;183:104-110. [PubMed: 23415494]

23. Hall DE, Arya S, Schmid KK, et al. Development and initial validation of the risk analysis index for measuring frailty in surgical populations. JAMA Surg. 2017;152:175-182. [PubMed: 27893030]

24. Bandeen-Roche K, Xue QL, Ferrucci L, et al. Phenotype of frailty: characterization in the women's health and aging studies. J Gerontol A Biol Sci Med Sci. 2006;61:262-266. [PubMed: 16567375]

25. Sousa-Santos AR, Amaral TF. Differences in handgrip strength protocols to identify sarcopenia and frailty - a systematic review. BMC Geriatr. 2017;17:238. [PubMed: 29037155]

26. Podsiadlo D, Richardson S. The timed "Up \& Go": a test of basic functional mobility for frail elderly persons. J Am Geriatr Soc. 1991;39:142-148. [PubMed: 1991946]

27. Malik AT, Quatman CE, Phieffer LS, Ly TV, Khan SN. Incidence, risk factors and clinical impact of postoperative delirium following open reduction and internal fixation (ORIF) for hip fractures: an analysis of 7859 patients from the ACS-NSQIP hip fracture procedure targeted database. Eur J Orthop Surg Traumatol. 2019;29:435-446. [PubMed: 30229446]

28. Racine AM, Fong TG, Gou Y, et al. Clinical outcomes in older surgical patients with mild cognitive impairment. Alzheimers Dement. 2018;14:590-600. [PubMed: 29190460]

29. Partridge JS, Dhesi JK, Cross JD, et al. The prevalence and impact of undiagnosed cognitive impairment in older vascular surgical patients. J Vasc Surg. 2014;60:1002-11.e3. [PubMed: 25017513]

30. Bozic KJ, Lau E, Kurtz S, Ong K, Berry DJ. Patient-related risk factors for postoperative mortality and periprosthetic joint infection in medicare patients undergoing TKA. Clin Orthop Relat Res. 2012;470:130-137. [PubMed: 21874391]

31. George EL, Arya S. The importance of incorporating frailty screening into surgical clinical workflow. JAMA Netw Open. 2019;2:e193538. [PubMed: 31074807]

32. Long LS, Shapiro WA, Leung JM. A brief review of practical preoperative cognitive screening tools. Can J Anaesth. 2012;59:798-804. [PubMed: 22638676]

33. Culley DJ, Flaherty D, Reddy S, et al. Preoperative cognitive stratification of older elective surgical patients: a Cross-Sectional Study. Anesth Analg. 2016;123:186-192. [PubMed: 27028776]

34. Subramaniam S, Aalberg JJ, Soriano RP, Divino CM. New 5-factor modified Frailty Index using American College of Surgeons NSQIP data. J Am Coll Surg. 2018;226:173-181.e8. [PubMed: 29155268]

35. Kenig J, Zychiewicz B, Olszewska U, Barczynski M, Nowak W. Six screening instruments for frailty in older patients qualified for emergency abdominal surgery. Arch Gerontol Geriatr. 2015;61:437-442. [PubMed: 26211706]

36. Joseph B, Zangbar B, Pandit V, et al. Emergency general surgery in the elderly: too old or too frail? J Am Coll Surg. 2016;222:805-813. [PubMed: 27113515] 
37. Winters AM, Hartog LC, Roijen H, Brohet RM, Kamper AM. Relationship between clinical outcomes and Dutch frailty score among elderly patients who underwent surgery for hip fracture. Clin Interv Aging. 2018;13:2481-2486. [PubMed: 30584288]

38. Drudi LM, Ades M, Mancini R, et al. Frailty assessment in older adults undergoing interventions for peripheral arterial disease. J Vasc Surg. 2019;70:1594-1602.e1. [PubMed: 31113723]

39. Makary MA, Segev DL, Pronovost PJ, et al. Frailty as a predictor of surgical outcomes in older patients. J Am Coll Surg. 2010;210:901-908. [PubMed: 20510798]

40. Pelavski AD, De Miguel M, Alcaraz Garcia-Tejedor G, et al. Mortality, geriatric, and nongeriatric surgical risk factors among the eldest old: a Prospective Observational Study. Anesth Analg. 2017;125:1329-1336. [PubMed: 28786844]

41. Andreou A, Lasithiotakis K, Venianaki M, et al. A comparison of two preoperative frailty models in predicting postoperative outcomes in geriatric general surgical patients. World J Surg. 2018;42:3897-3902. [PubMed: 30014293]

42. Wang HT, Fafard J, Ahern S, Vendittoli PA, Hebert P. Frailty as a predictor of hospital length of stay after elective total joint replacements in elderly patients. BMC Musculoskelet Disord. 2018;19:14. [PubMed: 29338705]

43. Parmar KL, Law J, Carter B, et al.; ELF Study Group. Frailty in older patients undergoing emergency laparotomy: results from the UK observational Emergency Laparotomy and Frailty (ELF) Study. Ann Surg. 2019 In press.

44. McGuckin DG, Mufti S, Turner DJ, Bond C, Moonesinghe SR. The association of peri-operative scores, including frailty, with outcomes after unscheduled surgery. Anaesthesia. 2018;73:819-824. [PubMed: 29569398]

45. Hewitt J, Moug SJ, Middleton M, Chakrabarti M, Stechman MJ, McCarthy K; Older Persons Surgical Outcomes Collaboration. Prevalence of frailty and its association with mortality in general surgery. Am J Surg. 2015;209:254-259. [PubMed: 25173599]

46. Chan S, Wong EKC, Ward SE, Kuan D, Wong CL. The predictive value of the clinical frailty scale on discharge destination and complications in older hip fracture patients. J Orthop Trauma. 2019;33:497-502. [PubMed: 31188261]

47. Donald GW, Ghaffarian AA, Isaac F, et al. Preoperative frailty assessment predicts loss of independence after vascular surgery. J Vasc Surg. 2018;68:1382-1389. [PubMed: 29773431]

48. Dasgupta M, Rolfson DB, Stolee P, Borrie MJ, Speechley M. Frailty is associated with postoperative complications in older adults with medical problems. Arch Gerontol Geriatr. 2009;48:78-83. [PubMed: 18068828]

49. Gleason LJ, Benton EA, Alvarez-Nebreda ML, Weaver MJ, Harris MB, Javedan H. FRAIL questionnaire screening tool and short-term outcomes in geriatric fracture patients. J Am Med Dir Assoc. 2017;18:1082-1086. [PubMed: 28866353]

50. McIsaac DI, Moloo H, Bryson GL, van Walraven C. The Association of frailty with outcomes and resource use after emergency general surgery: a Population-Based Cohort Study. Anesth Analg. 2017;124:1653-1661. [PubMed: 28431425]

51. McIsaac DI, Bryson GL, van Walraven C. Association of frailty and 1-year postoperative mortality following major elective noncardiac surgery: a Population-Based Cohort Study. JAMA Surg. 2016;151:538-545. [PubMed: 26791334]

52. Esses G, Andreopoulos E, Lin HM, Arya S, Deiner S. A comparison of three frailty indices in predicting morbidity and mortality after on-pump aortic valve replacement. Anesth Analg. 2018;126:39-45. [PubMed: 28857797]

53. Aizpuru M, Staley C, Reisman W, Gottschalk MB, Schenker ML. Determinants of length of stay after operative treatment for femur fractures. J Orthop Trauma. 2018;32:161-166. [PubMed: 29558372]

54. Panayi AC, Orkaby AR, Sakthivel D, et al. Impact of frailty on outcomes in surgical patients: a systematic review and meta-analysis. Am J Surg. 2019;218:393-400. [PubMed: 30509455]

55. Bellamy JL, Runner RP, Vu CCL, Schenker ML, Bradbury TL, Roberson JR. Modified frailty index is an effective risk assessment tool in primary total hip arthroplasty. J Arthroplasty. 2017;32:2963-2968. [PubMed: 28559198] 
56. Arya S, Long CA, Brahmbhatt R, et al. Preoperative frailty increases risk of nonhome discharge after elective vascular surgery in home-dwelling patients. Ann Vasc Surg. 2016;35:19-29. [PubMed: 27263810]

57. Karam J, Tsiouris A, Shepard A, Velanovich V, Rubinfeld I. Simplified frailty index to predict adverse outcomes and mortality in vascular surgery patients. Ann Vasc Surg. 2013;27:904-908. [PubMed: 23711971]

58. Hall DE, Arya S, Schmid KK, et al. Association of a frailty screening initiative with postoperative survival at 30, 180, and 365 days. JAMA Surg. 2017;152:233-240. [PubMed: 27902826]

59. van der Windt DJ, Bou-Samra P, Dadashzadeh ER, Chen X, Varley PR, Tsung A. Preoperative risk analysis index for frailty predicts short-term outcomes after hepatopancreatobiliary surgery. HPB (Oxford). 2018;20:1181-1188. [PubMed: 30005992]

60. Isharwal S, Johanning JM, Dwyer JG, Schimid KK, LaGrange CA. Preoperative frailty predicts postoperative complications and mortality in urology patients. World J Urol. 2017;35:21-26. [PubMed: 27172940]

61. Melin AA, Schmid KK, Lynch TG, et al. Preoperative frailty risk analysis index to stratify patients undergoing carotid endarterectomy. J Vasc Surg. 2015;61:683-689. [PubMed: 25499711]

62. Bandeen-Roche K, Seplaki CL, Huang J, et al. Frailty in older adults: a nationally representative profile in the United States. J Gerontol A Biol Sci Med Sci. 2015;70:1427-1434. [PubMed: 26297656]

63. Suskind AM, Finlayson E. A call for frailty screening in the preoperative setting. JAMA Surg. 2017;152:240-241. [PubMed: 27902810]

64. Walston J, Fedarko N, Yang H, et al. The physical and biological characterization of a frail mouse model. J Gerontol A Biol Sci Med Sci. 2008;63:391-398. [PubMed: 18426963]

65. Deepa SS, Bhaskaran S, Espinoza S, et al. A new mouse model of frailty: the $\mathrm{Cu} / \mathrm{Zn}$ superoxide dismutase knock-out mouse. Geroscience. 2017;39:187-198. [PubMed: 28409332]

66. Pritchard JM, Kennedy CC, Karampatos S, et al. Measuring frailty in clinical practice: a comparison of physical frailty assessment methods in a geriatric out-patient clinic. BMC Geriatr. 2017;17:264. [PubMed: 29132301]

67. Gurlit S, Gogol M. Prehabilitation is better than cure. Curr Opin Anaesthesiol. 2019;32:108-115. [PubMed: 30507681]

68. Walston J, Robinson TN, Zieman S, et al. Integrating frailty research into the medical specialtiesreport from a U13 conference. J Am Geriatr Soc. 2017;65:2134-2139. [PubMed: 28422280]

69. Rockwood K, Silvius JL, Fox RA. Comprehensive geriatric assessment. Helping your elderly patients maintain functional well-being. Postgrad Med. 1998;103:247-249, 254.

70. Solomon DH. Geriatric assessment: methods for clinical decision making. JAMA. 1988;259:24502452. [PubMed: 3280847]

71. Gill TM, Baker DI, Gottschalk M, et al. A prehabilitation program for physically frail communityliving older persons. Arch Phys Med Rehabil. 2003;84:394-404. [PubMed: 12638108]

72. Mohile SG, Xian Y, Dale W, et al. Association of a cancer diagnosis with vulnerability and frailty in older medicare beneficiaries. J Natl Cancer Inst. 2009;101: 1206-1215. [PubMed: 19638506]

73. Ethun CG, Bilen MA, Jani AB, Maithel SK, Ogan K, Master VA. Frailty and cancer: implications for oncology surgery, medical oncology, and radiation oncology. CA Cancer J Clin. 2017;67:362377. [PubMed: 28731537]

74. Friedman J, Lussiez A, Sullivan J, Wang S, Englesbe M. Implications of sarcopenia in major surgery. Nutr Clin Pract. 2015;30:175-179. [PubMed: 25681482] 

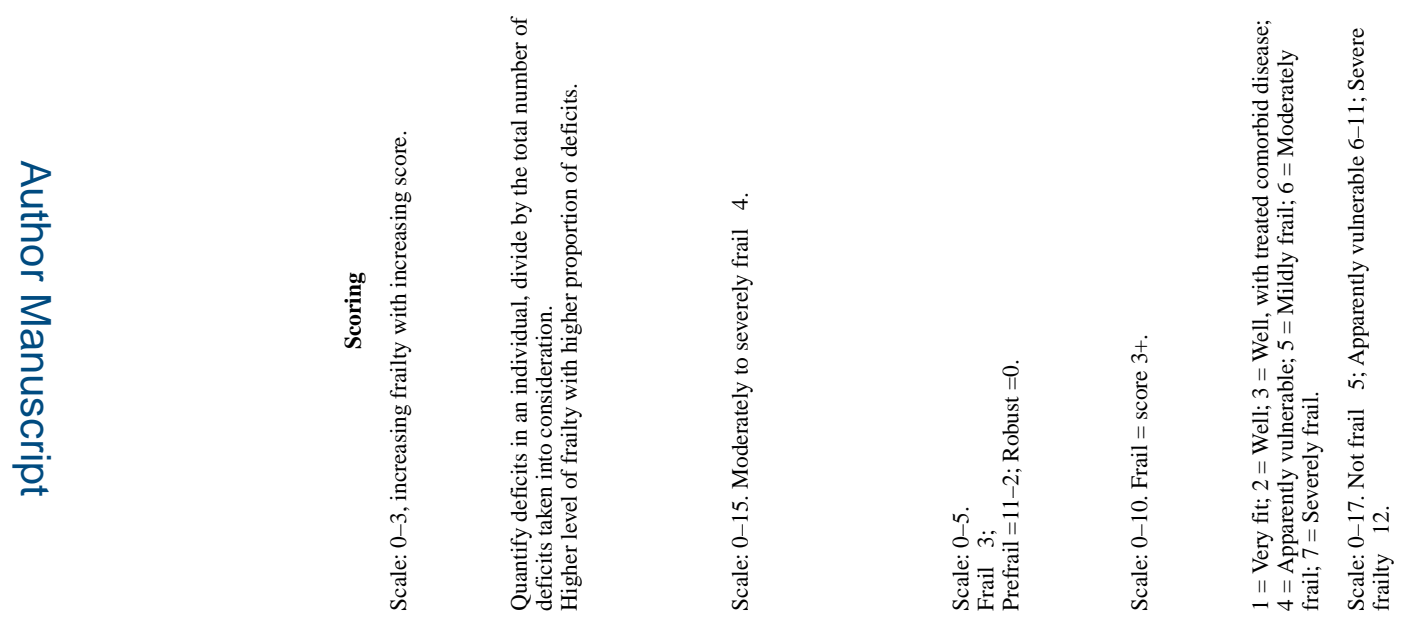

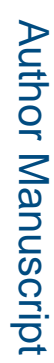

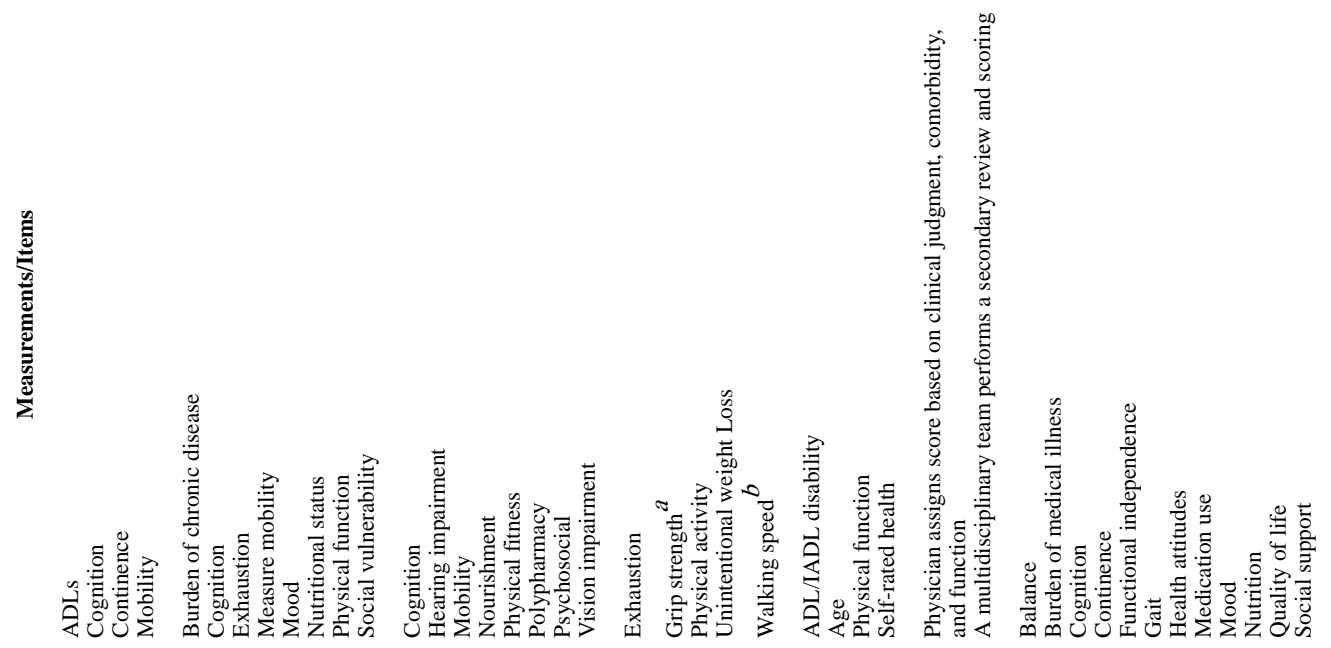

西

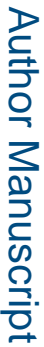

मे

ב

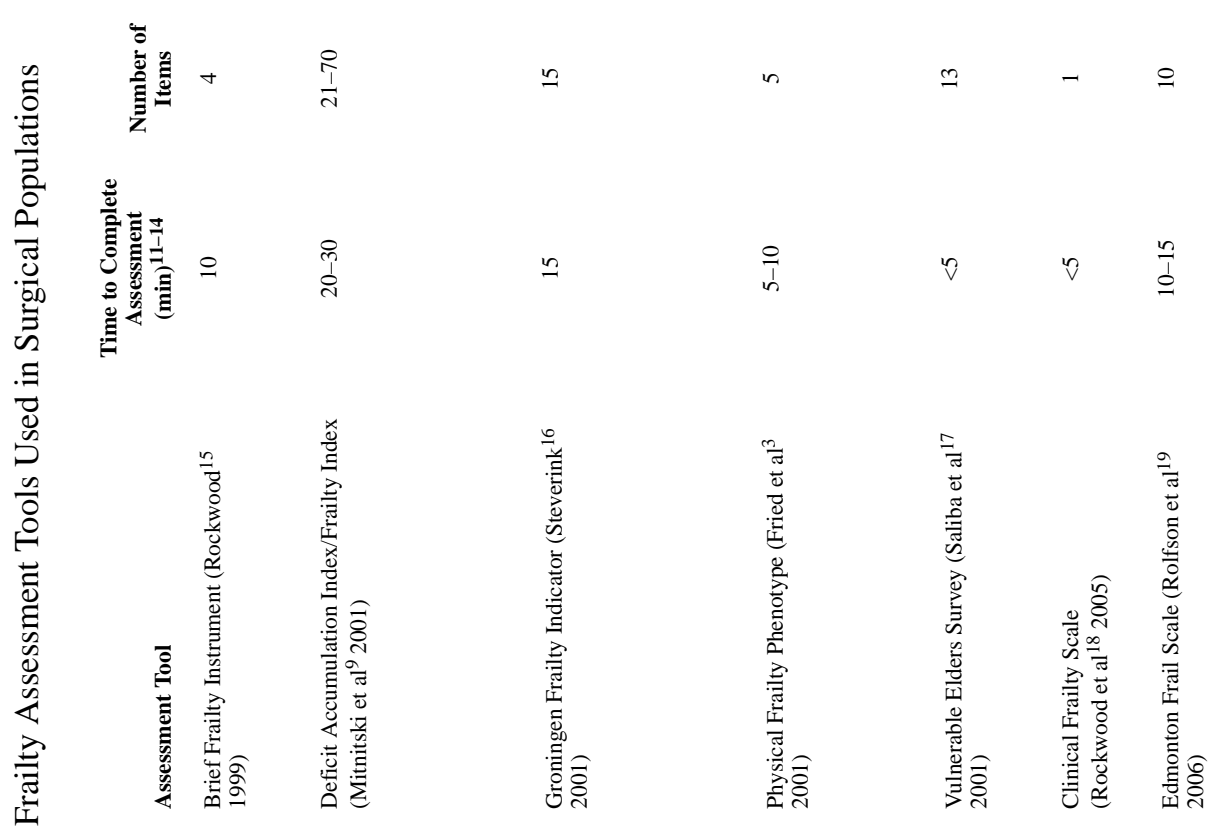

Anesth Analg. Author manuscript; available in PMC 2020 July 15. 


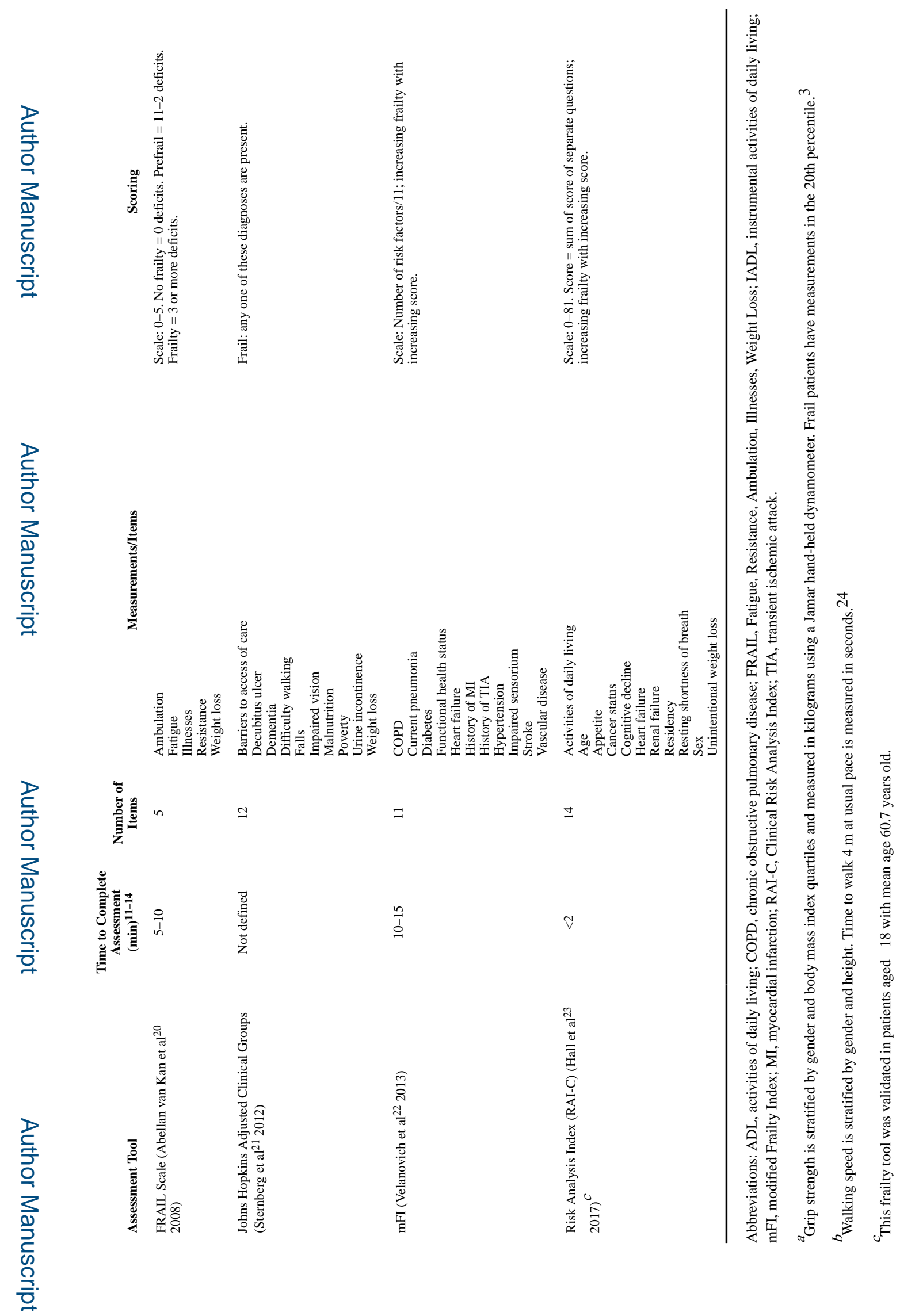

Anesth Analg. Author manuscript; available in PMC 2020 July 15. 

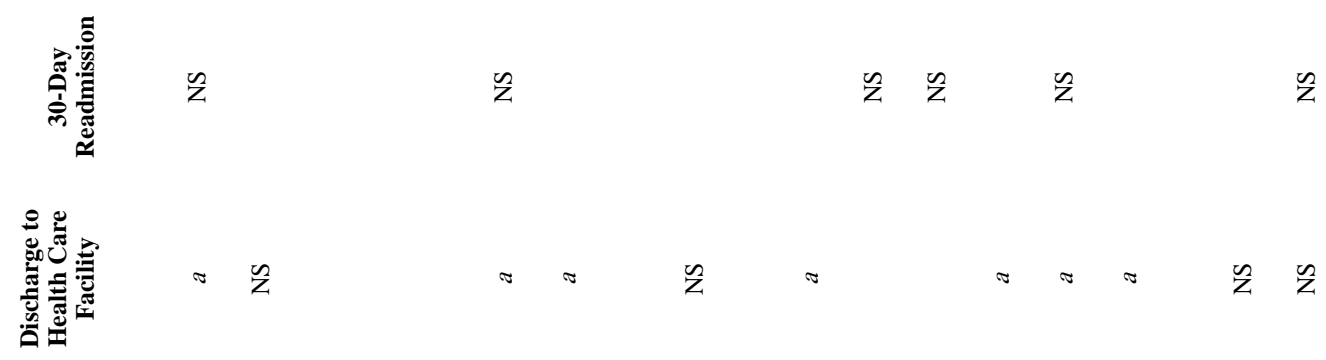

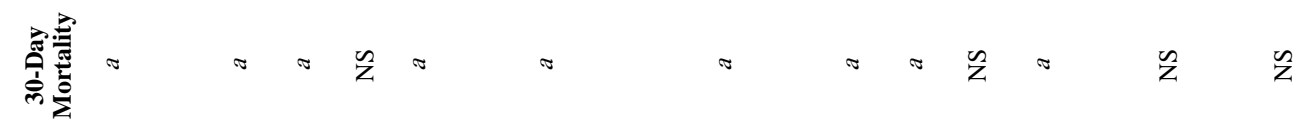

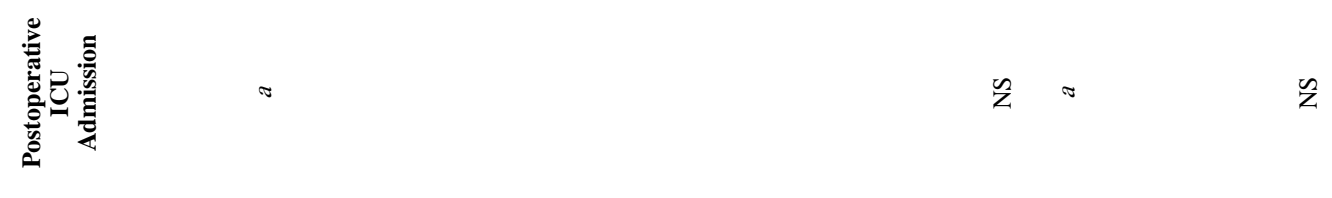

롤

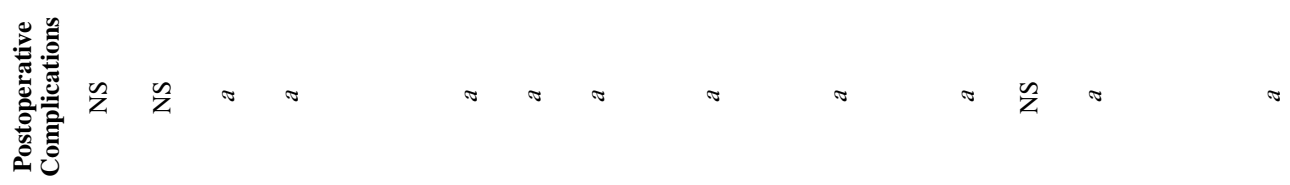

$\frac{\text { ก }}{\frac{0}{0}}$

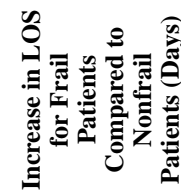

竎

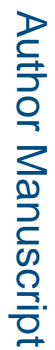

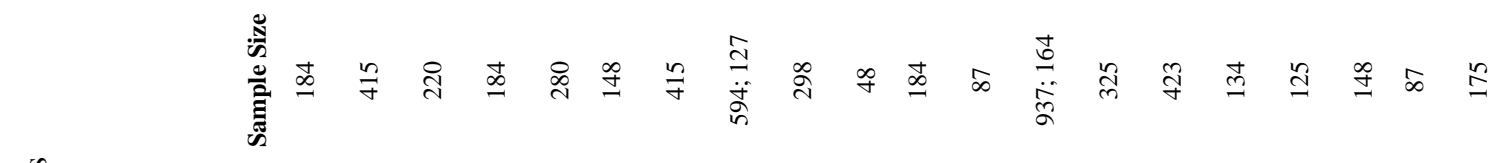

로을
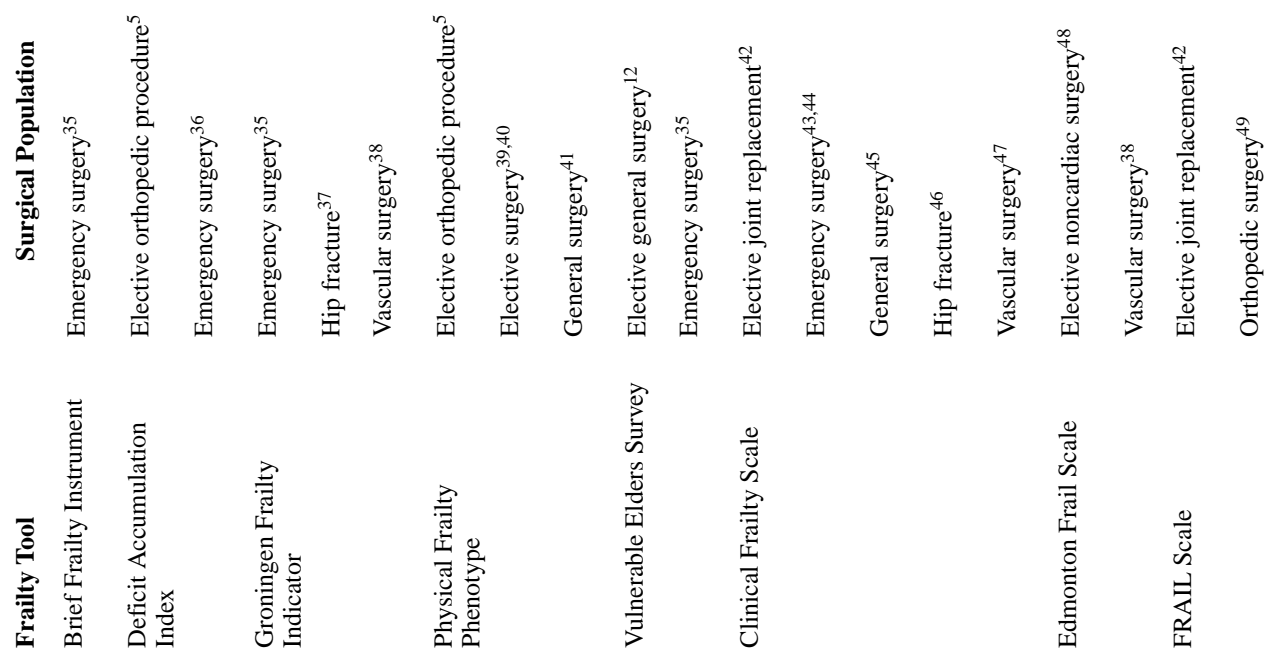

Anesth Analg. Author manuscript; available in PMC 2020 July 15. 


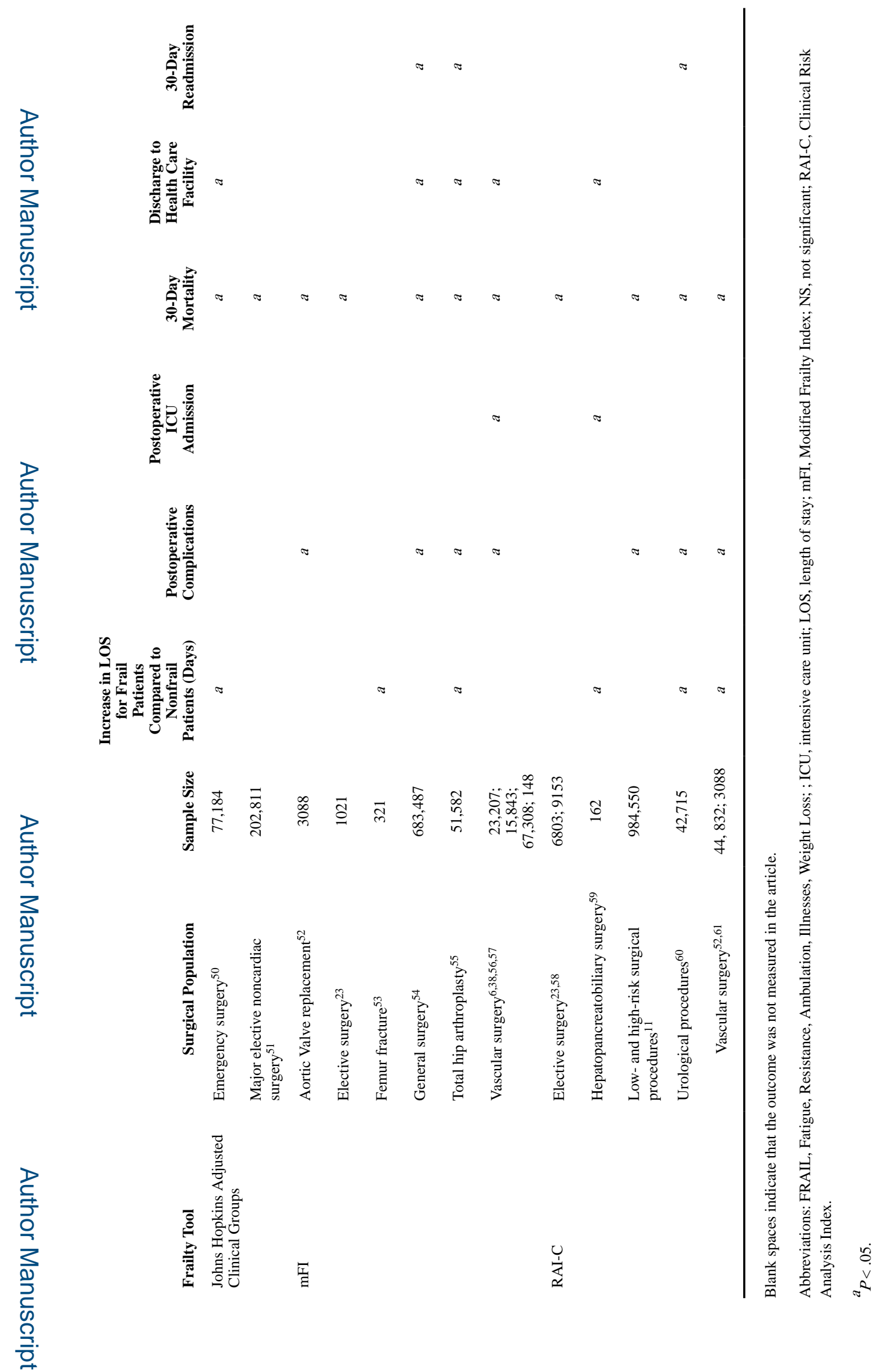

Anesth Analg. Author manuscript; available in PMC 2020 July 15. 